\title{
Effects of Age on the Generalization and Incubation of Memory in the F344 Rat
} Frank P. Houston, ${ }^{4}$ Gail D. Stevenson, ${ }^{4}$ Bruce L. McNaughton, ${ }^{1,3,4}$ and Carol A. Barnes ${ }^{1,2,4,5}$

Departments of ${ }^{1}$ Psychology, ${ }^{2}$ Neurology, and ${ }^{3}$ Physiology and ${ }^{4}$ Arizona Research Laboratories Division of Neural Systems, Memory, and Aging

University of Arizona

Tucson, Arizona 85724 USA

\section{Abstract}

Freezing (immobility) in the presence of aversive stimuli is a species-specific behavior that is used as an operational measure of fear. Conditioning of this response to discrete sensory stimuli and environmental context cues has been used as a tool to study the neuropsychology of memory dynamics and their development over the lifespan. Three age groups of F344 rats $(3,9$, and 27 month) received tone-foot shock pairing (or tone only) in a distinctive chamber on two consecutive days. Separate subgroups of rats from each age group were then tested, at retention intervals of 1,20 , 40 , or 60 days, for context-mediated fear in the environment in which they were trained, for generalization of the fear response to a novel chamber, and for fear of the tone. Beginning at day 20 , the 27-month-old rats exhibited less freezing behavior than did younger rats when tested in the conditioning context. This age difference was a result of freezing behavior becoming progressively stronger with time in the two younger age groups, a phenomenon that has been referred to as memory incubation. Incubation of the contextual fear response was not detected in the old rats. In a novel context, all age groups exhibited significantly more freezing than did control animals. There was also pronounced incubation of this generalized freezing response, and the extent of

${ }^{5}$ Corresponding author. incubation declined significantly with age. In the novel context, the freezing response to the tone was robust in all age groups and increased over time, in constant proportion to the degree of freezing elicited by the novel context itself, prior to tone onset. The fact that old animals are known to be relatively selectively impaired in forms of memory that depend on a functional hippocampus suggests a possible explanation for the reduced incubation effects seen in old rats; however, whether the increased expression of fear over time is mediated by a hippocampal-dependent memory consolidation process or whether it reflects a generalized increase in the gain of the circuitry mediating the fear response itself, remains to be determined.

\section{Introduction}

For effective learning to occur, information acquired during training or conditioning must be processed and stored appropriately. Both the hippocampus and neocortex are essential to acquisition and subsequent storage of information about specific experiences, and the interaction between these structures appears to be required for the consolidation of such memories into the long-term, general knowledge base of the animal (e.g., McGaugh 1966; Marr 1971; Eichenbaum et al. 1994; Myers and Gluck 1994; Squire et al. 1984; McClelland et al. 1995; Squire and Alvarez 1995; Buzsáki 1996; Knowlton and Fanselow 1998). The nature of the hippocampal-neocortical interaction in the memory consolidation process has not yet been established clearly; however, there are several

LEARNING \& MEMORY 6:111-119 @ 1999 by Cold Spring Harbor Laboratory Press ISSN1072-0502/99 \$5.00

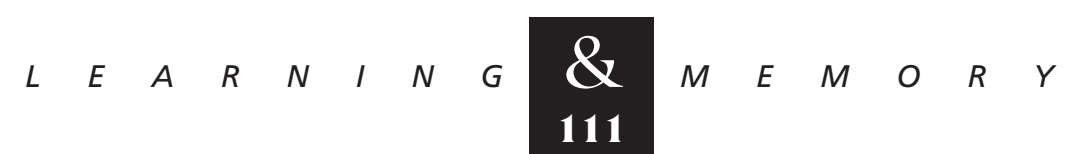


overlapping theories about the process of memory consolidation and the role of the hippocampus in it.

One of the earlier ideas about the consolidation process is that the hippocampus provides an input to the neocortex that, in some unspecified way, acts to bind together the components of episodic memory traces (e.g., Squire 1992). More computationally oriented theories include Marr's idea that the hippocampus acts as a simple event memory or autoassociator (Marr 1971). He proposed that during periods when the brain is not actually processing external inputs (e.g., sleep), these memories might be reactivated spontaneously and cause reactivation of the same regions of neocortex from which the stored pattern was originally transmitted. Marr (1971) suggested that this would result in an opportunity for the neocortex to form new categories and event classes. Recent neurophysiological studies have confirmed that during sleep and quiet wakefulness, there is some form of reactivation, in both hippocampus and neocortex, of neural ensemble activity patterns corresponding to recent events (Pavlides and Winson 1989; Wilson and McNaughton 1994; Skaggs and McNaughton 1996; Qin et al. 1997). A somewhat similar notion to that of Marr's was expressed by Teyler and DiScenna (1986) in their hippocampal indexing theory, although they did not explicitly discuss the possible role of hippocampal "index codes" in the memory consolidation process. McClelland et al. (1995) developed an explicit computational theory for why it is important for hippocampus and neocortex to interact in the consolidation process. The core of their hypothesis focuses on the phenomenon of catastrophic interference in the training of connectionist networks (McCloskey and Cohen 1989) and its solution through the repeated interleaving of the learning set items, coupled with small weight changes. According to McClelland et al. (1995), the reason why the neocortex would be susceptible to catastrophic interference is that it must appropriately generalize and categorize experiences using some form of gradient descent learning. Gradient descent requires a repetitive, interleaved training regimen, and it was proposed that the hippocampus could serve as a source of repeated, "virtual" events as training trials for the neocortical circuitry. Such ideas on the consolidation process consider the hippocampus to have a time-limited role in memory storage. An alternative view, proposed by Nadel and Moscovitch (1997, 1998), is that the hippocampus plays a more permanent role in episodic memory storage; however, they do not dispute the probable importance of interactions between hippocampus and neocortex over long periods following the initial registration of information.

A number of studies have shown that lesions to the hippocampus in rats, rabbits, and nonhuman primates result in a temporally graded amnesia for previously learned material (Winocur 1990; ZolaMorgan and Squire 1990; Kim and Fanselow 1992; Bolhuis et al. 1994; Kim et al. 1995; Maren and Fanselow 1997; Anagnostaras et al. 1999; R. Sutherland, K. Arnold, and A. Rodriguez, unpubl.) that may be similar to the retrograde amnesia for events that can occur in humans with damage to the hippocampal region (e.g., Scoville and Milner 1957; Milner 1972; Squire 1992). For example, in rats, the hippocampus appears to be necessary for the expression of the freezing response to the context of the shock environment (e.g., Phillips and Le Doux 1992; Maren et al. 1997; McNish et al. 1997; Aguado et al. 1998; Good et al. 1998), even in the absence of the explicit conditioned stimulus (CS), for several weeks following initial training (Kim and Fanselow 1992; Anagnostaras et al. 1999). Alterations in hippocampus-dependent memory dynamics over periods of a few days to weeks occur naturally during the aging process (e.g., Barnes 1990; Rapp and Gallagher 1997). This study was designed to examine how aging affects the longterm reorganization and possible decay of memories that initially depend on an intact hippocampus, after the period in which the hippocampus is normally presumed no longer to be essential for their expression. As with some other forms of hippocampus-independent memory, tone-shock associations appear to be acquired equally well by young and old rats (Oler and Markus 1998; E.J. Brown, K. Hunt, and D.J. Markus, unpubl.; but see Stoehr and Wenk 1995). Both hippocampal-dependent and hippocampal-independent memories were assessed using distributed, Pavlovian fear conditioning methods similar to those described by Kim and Fanselow (1992). To avoid possible reinstatement effects that may have been present in previous studies of age effects (e.g., Oler and Markus 1998), retention testing was conducted in independent groups of animals at time intervals out to 61 days. Some of these data have been published in abstract form (F.P. Houston, G.D. Stevenson, B.L. McNaughton, and C.A. Barnes, unpubl.).

$$
\begin{array}{lllllllllllllll} 
& E & A & R & N & I & N & G & \boldsymbol{Q} \\
\mathbf{1 1 2} & M & E & M & O & R & Y
\end{array}
$$




\section{Materials and Methods}

\section{SUBJECTS}

A total of 144 male $\mathrm{F} 344$ rats were used in these experiments. All rats were obtained from the National Institute on Aging Charles River colony at 3, 9, or 27 months of age. The experiment was conducted in three phases. The first set contained 37,38 , and 39 rats in the respective age groups; the second set contained 5 and 8 rats at 9 and 27 months, respectively; and the third set contained 6, 6, and 5 rats at 3,9, and 27 months of age, respectively. All rats were handled, given health checks, and were individually housed in $45 \times 24 \times 21-\mathrm{cm}$ transparent Plexiglas cages at the beginning of the experiment. The rats were maintained on a reversed 12:12 hour light/dark cycle (lights off at 10 a.m.) and given food and water on an ad libitum schedule. All rats were handled for 1-2 min/day in the week before behavioral testing began, and 10-20 $\mathrm{min} /$ week after that until completion of the study. One week prior to the initiation of the conditioning experiment, all animals underwent training in the spatial version of the Morris (1984) swim task (data not shown). As established in many previous studies (e.g., Gage et al. 1984; Gallagher et al. 1993; Shen and Barnes 1996) there was a significant decline with age in performance of this task.

\section{CONDITIONING AND NOVEL APPARATI}

The conditioning apparatus consisted of a $28 \times 21 \times 22-\mathrm{cm}$ operant chamber (Lafayette Instruments Co., North Lafayette, IN). The floor of the chamber was made of 18 stainless steel rods that were wired to a shock generator and scrambler for the delivery of shock. This chamber was used for initial acquisition conditioning and once again for each rat (without shock) at a specified time interval following training, to test for behavioral freezing to this context (Fig. 1A). A computer was used to control the tone delivery and shock at the appropriate time intervals. A video camera recorded the entire conditioning session, with the beginning and end of the session signaled by a diode (not visible to the rat) mounted next to the chamber. The novel apparatus was housed in a separate room and consisted of a $30-\mathrm{cm}$-diameter Plexiglas cylinder tube placed vertically on the surface of a metal table (Fig. 1B). A $20 \times 20$-cm mirror was placed behind the animal (outside the chamber), and these sessions were also videorecorded.
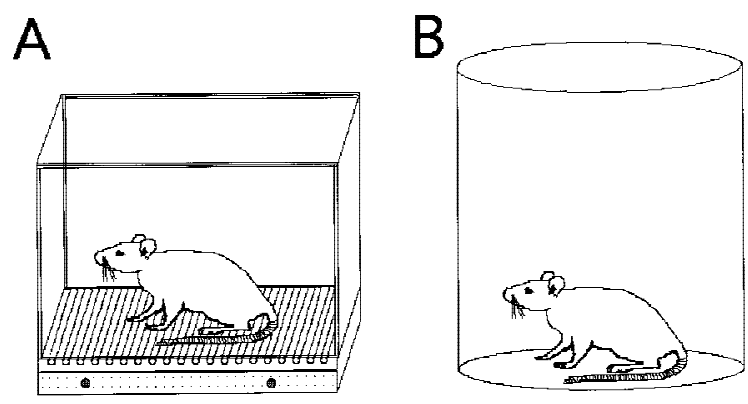

Figure 1: Schematic diagram of the conditioning chamber $(A)$ in which the experimental groups received tone-foot-shock pairings, and the novel chamber $(B)$ in which context generalization and conditioning to the tone were tested. The two chambers were located in different rooms.

\section{CONDITIONING PROCEDURE}

All rats in the conditioning groups received 2 sessions of either 8 (rats in set 1) or 4 (rats in set 2 and 3) tone-foot-shock pairings separated by 3-min periods. Each session was initiated by a 3-min "exploration" period prior to the first shock, and these two sessions were separated by $\sim 12 \mathrm{hr}$. For all rats, the tone and shock parameters of this conditioning were tone $=2 \mathrm{kHz}, 90 \mathrm{~dB}, 30 \mathrm{sec}$; foot shock $=1$ $\mathrm{mA}, 60 \mathrm{~Hz}, 2 \mathrm{sec}$. A subset of rats in set 2 and 3 also included animals that were never shocked $(n=2$, 5 , and 5 in the 3,9, and 27-month groups, respectively), but were tested for their freezing behavior to the conditioning apparatus and to the tone at intervals corresponding to those in which the conditioned animals were tested (with the exception of the 60-day time point). Separate groups of rats that went through the conditioning procedure in the three age groups were tested in the original conditioning apparatus at either 1, 20, 40, or 60 days after the last conditioning day. No tone or shock was delivered during the $8 \mathrm{~min}$ of retesting. Following this test the rat was returned to his cage and the apparatus was cleaned using dilute (5\%) ammonium hydroxide. All rats were initially randomly assigned to the testing intervals; however, 8 of the 52 old animals were moved to earlier testing days because of a decline in overall health. Because the mean performance scores of these old rats was not statistically different from the randomly assigned animals $(P>0.05)$, their data were included in the analysis.

Testing in the novel apparatus was conducted one day after testing in the original conditioning context (days 2, 21, 41, 61) in a different room.

$$
\begin{array}{lllllllllllllll}
L & E & A & R & N & I & N & G & \underset{113}{\mathbf{Z}} & M & E & M & O & R & Y
\end{array}
$$


Rats were individually placed in the novel apparatus for a total of $11 \mathrm{~min}-3 \mathrm{~min}$ of silence followed by $8 \mathrm{~min}$ of continuous tone. After testing with the tone in this novel environment, the animal was returned to its cage and the apparatus was cleaned with a dilute (5\%) acetic acid solution.

\section{SENSORY AND PAIN THRESHOLD TESTS}

At the end of the experiment, rats were placed back into the conditioning chamber to test for sensory and pain thresholds. The intensity was increased from $0.1 \mathrm{~mA}$ upwards until the rat appeared to detect the stimulus by raising its foot. If the rat raised its foot to a second stimulus of that amplitude, then this stimulus amplitude was defined as the "threshold intensity." The intensity of the stimulus was then increased to the point at which the rat quickly removed at least one foot from the grid. This was defined as the "pain threshold."

\section{DATA ANALYSIS}

The videotaped behavior was scored off-line at 5-sec intervals simultaneously by two observers (the correlation between observers was 0.64). The primary dependent variable used for this experiment was the amount of time spent freezing. Freezing was defined as the lack of all movement except those movements necessary for respiration. The data analyzed for the conditioning experiment included the percent time spent in movement for the 3 min before the first conditioning session, the 3 min before the second conditioning session, the first $3 \mathrm{~min}$ of the retest in the context, the first 3 min of the test in the novel environment before tone, and the first $3 \mathrm{~min}$ during tone presentation in the novel environment. The data were analyzed using one or two-way analyses of variance (ANOVAs), with $\alpha$ set at the 0.05 level. Fischer PLSD (protected least significant difference) tests were used for post-hoc analyses.

\section{Results}

\section{SENSORY AND PAIN THRESHOLD TESTS}

There was a significant effect of age in sensation threshold $\left(F_{2,111}=4.03, P \leq 0.02\right)$. Post hoc tests revealed that young and adult rats did not differ from one another $(P>0.05)$ but that old rats had a significantly lower threshold than did the young $(P \leq 0.03)$ and adult $(P \leq 0.01)$ rats. There was a similar significant effect of age in the pain threshold $\left(F_{2,111}=6.99, P \leq 0.002\right)$, with old rats showing a significantly lower threshold than did the young $(P \leq 0.03)$ or adult rats $(P \leq 0.0003)$. The young and adult rats did not differ significantly. Because the largest difference between groups was only $\sim 0.05 \mathrm{~mA}$, the direction of the difference did not covary with chronological age and the conditioning shock was more than twice the sensory threshold, the shock parameters were considered appropriate for this age comparison.

\section{CONDITIONED RESPONSE TESTS IN EXPERIMENTAL AND CONTROL RATS}

Figure $2 \mathrm{~A}$ shows the percent movement for all 144 animals during the $3 \mathrm{~min}$ before conditioning commenced. Movement for all animals was high and all values approached $100 \%$. There was no significant effect of age $\left(F_{2,138}=1.043, P>0.05\right)$ or of treatment $\left(F_{1,138}=1.247, P>0.05\right)$. During the initial 3-min period prior to the second set of conditioning trials, dramatic decreases in movement were observed for all age groups of experimental animals, whereas high levels of movement were observed for all control animals (Fig. 2B). There was no significant effect of age $\left(F_{2,138}=1.020\right.$, $P>0.05)$, but there was a significant effect of treatment $\left(F_{1,138}=45.84, P \leq 0.0001\right)$. At the 1-day retention interval, the control animals again showed high levels of movement, whereas minimal movement was observed in the conditioned animals (Fig. 2C). Because of the small number of control animals $(n=12)$ at the $1-, 20-$, and 40-day time intervals, we pooled all intervals for both control and experimental rats to enable a treatment comparison for the 3 age groups. This resulted in a significant effect of treatment $\left(F_{1,111}=286.1\right.$, $P \leq 0.0001)$ but no effect of age $\left(F_{2,111}=2.922\right.$, $P>0.05)$. Thus, the behavior of the control rats in all age groups was significantly different from the behavior of the rats that received the conditioning treatment.

\section{RETENTION OF THE CONTEXT IN WHICH CONDITIONING TOOK PLACE}

Figure 3 (left panels) illustrates the behavior of the animals during the first $3 \mathrm{~min}$ in the conditioning apparatus (no tone, no shock). The fraction of time spent in motion did not return to precondi- 
A
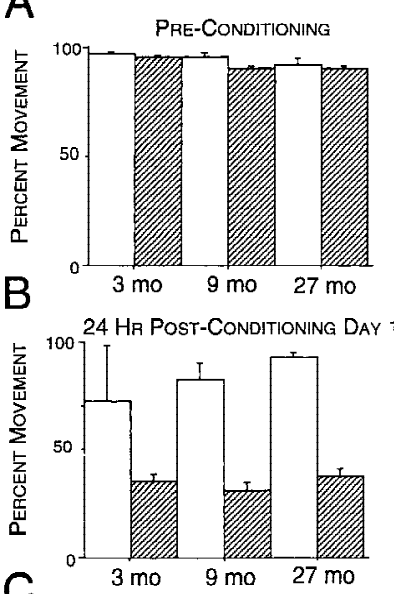

24 HR POST-CONDITIONING DAY 2

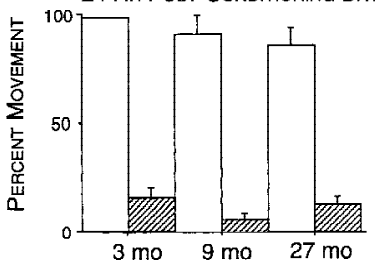

Figure 2: $(A)$ Mean percent movement in the conditioning chamber during the first $3 \mathrm{~min}$ before training began for the conditioned rats (hatched bars) and for the control rats (open bars). The amount of movement in the chamber was high and not different between treatment or age groups before conditioning procedures were initiated. (B) Mean percent movement 3 min before the second set of conditioning trials were initiated on the second day of training for the experimental rats (shaded bars) and for the control rats (open bars) that did not receive shock in the conditioning environment the previous day. The experimental rats moved significantly less than did the control rats in each of the 3 age groups. (C) Mean percent movement $24 \mathrm{hr}$ following the final conditioning treatment for the Day 1 group of experimental rats vs. control rats. No tone or shock was presented on the Day 1 retention test or on subsequent retention test days. Control rats again spent significantly more time moving than did the experimental rats, and at this time interval there was no difference between age groups in freezing behavior for the conditioning context.

tioning levels of $>90 \%$ for rats in any of the 3 age groups over the 60 days of testing. However, the behavior was different between the three age groups over time. There was a significant effect of age $\left(F_{2,132}=3.242, P \leq 0.04\right)$ but no significant effect of retention interval $\left(F_{3,132}=2.537\right.$, $P>0.05)$. The overall effect of retention interval was not significant in part because the old rats did not show a consistent change in behavior over time. Figure 3 (left panels) illustrates that the young and adult animals showed increased freezing behavior (less movement) over the 60-day retention interval (young: slope $=-0.0021, r^{2}=0.838$; adult: slope $=-0.0007, r^{2}=0.876$ ), whereas the slope for fractional movement in old rats did not exhibit a significant linear trend (old: slope $=+0.0004, r^{2}=0.035$ ). Because the old rats did not increase their freezing behavior over time, the old rats showed statistically more movement on days 20 through 60 than did than the young and adult rats $(P \leq 0.001$ and $P \leq 0.0001$, respectively).

\section{GENERALIZATION OF FREEZING BEHAVIOR TO THE NOVEL CONTEXT}

Figure 3 (middle panels) shows that freezing behavior over the first $3 \mathrm{~min}$ in the novel chamber (before the tone was initiated) was intermediate between preconditioning levels (Fig. 2A) and contextual retesting (Fig. 3, left). There was a significant effect of age $\left(F_{2,132}=8.347, P \leq 0.0005\right)$, a significant effect of retention interval $\left(F_{3,132}\right.$ $=16.24, P \leq 0.0001)$ and an age-by-retention interval interaction $\left(F_{6,132}=2.545, P \leq 0.023\right)$. The significant effect of retention interval was attributable to all age groups showing increased freezing behavior over time. The interaction can be explained by the reduction in the slope of the percent movement versus interval function at progressively older ages (young: slope $=-0.009, r^{2}=0.822$; adult: slope $=-0.004, r^{2}=0.590 ;$ old: slope $=-0.0025$, $\left.r^{2}=0.830\right)$.

\section{RETENTION OF THE TONE RESPONSE IN THE NOVEL CHAMBER}

Figure 3 (right panels) illustrates that when the tone was delivered (with no shock) in the novel chamber, all age groups exhibited substantial freezing during the 3-min period measured, at all time intervals. There was no effect of age $\left(F_{2,132}\right.$ $=0.381, \quad P>0.05)$, but there was a significant effect of retention interval $\left(F_{3,132}=6.015\right.$, $P \leq 0.0008$ ), which was due to the fact that all rats, in this case, exhibited significant increases in freezing behavior in response to the tone over 61 days. Slope analysis for the young, adult, and old animals revealed similar trends for decreased movement over the 60-day testing period (young: slope $=-0.0028, r^{2}=0.980$; adult: slope $=-0.0011$, $r^{2}=0.770$; old: slope $\left.=-0.0010, r^{2}=0.731\right)$. The

$$
\begin{array}{lllllllllllllll} 
& E & A & R & N & I & N & G & \boldsymbol{Q} \\
\mathbf{1 1 5} & M & E & M & O & R & Y
\end{array}
$$


Figure 3: Mean percent movement in separate groups of young, adult, and old rats tested at 4 time intervals $(1,20,40$, and 60 days for context, and 2, 21, 41, 61 days for novel and tone conditions) after conditioning training. During these retention tests the rats were not given shock. The first test was in the conditioning chamber (context, first column), the second on the following day in the novel chamber (second column), and the tone was tested in the novel chamber (third column) immediately following the 3-min baseline test in the novel chamber. All rats showed significant levels of freezing behavior in the conditioning context out to 60 days (first column). The young and adult rats showed increased freezing with time, or an "incubation" effect, which was not apparent in the old rats. The first time the rats were placed into the novel chamber (second column), they all showed significantly less
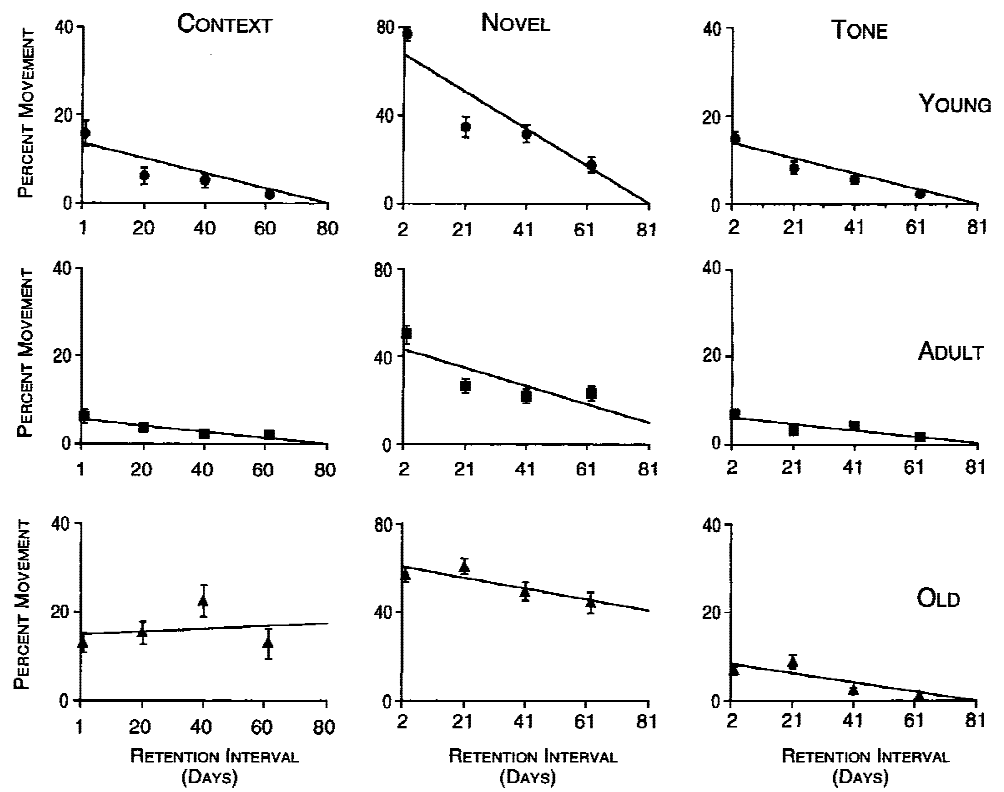

movement than before training (Fig. 2A), but significantly more movement than in the conditioning context. Note that the scale for the novel condition is twice that of the context or tone conditions. This suggests that the freezing response generalized to this novel context and that this generalization showed incubation over the retention interval. The amount of increased movement suppression was greatest in the youngest rats and least in the old rats. All rats showed strong movement suppression when the tone was sounded in the novel context, and there were no differences between age groups (third column).

question arises as to whether the decrease in movement over retention interval can be attributed to a true change in sensitivity to the tone itself or whether the increased tone response is related in some way to the increased response to the novel context. The percent decrement in movement following the tone compared to the movement baseline during the three minutes in the novel chamber before the tone (i.e., Fig. 3, left panels), was calculated as $(\mathrm{Mn}-\mathrm{Mt}) / \mathrm{Mn}(\times 100)$, where $\mathrm{Mn}$ and $\mathrm{Mt}$ are the movement measures for the novel only and tone epochs. The percent decrement in movement caused by the tone maintained an essentially constant proportion across retention intervals to the freezing response to the novel context only (Fig. 4).

\section{Discussion}

The overall pattern of results can be summarized as follows: (1) All age groups exhibited robust conditioned suppression of movement in response to both the tone and the conditioning context relative to control rats. (2) Suppression of movement to the original context in the absence of tone or shock increased significantly over retention interval in the young and adult animals, but not in the old. The poor confidence interval for the slope estimate for the old rat data preclude a decision as to whether the actual slope was slightly positive, zero, or negative. (3) For all rats, suppression of movement in the novel context in the absence of the tone was significantly greater than in control animals and also increased significantly over retention interval. The relative decrease in movement was significant in all age groups, but this incubation effect was progressively smaller with age. (4) The absolute magnitude of movement suppression attributable to the tone increased over retention interval, in a manner that was proportional to the degree of suppression that could be attributed to the novel context itself.

The incubation process is a progressive increase in the measure of memory strength with time following some aversive stimulus, without further exposure to the stimulus itself (McAllister and McAllister 1967; Eysenck 1968). Incubation is hypothesized to accompany the memory consolidation process, not only for hippocampal-dependent aversive learning experiences, but also in cases of perceptual learning (Karni et al. 1994). Gabriel and Vogt (1972) suggested that incubation is a compensatory process that mitigates memory loss that would naturally occur as time progresses, by en-

$$
\begin{array}{lllllllllllllll} 
& E & A & R & N & I & N & G & \boldsymbol{Q} \\
\mathbf{1 1 6} & M & E & M & O & R & Y
\end{array}
$$




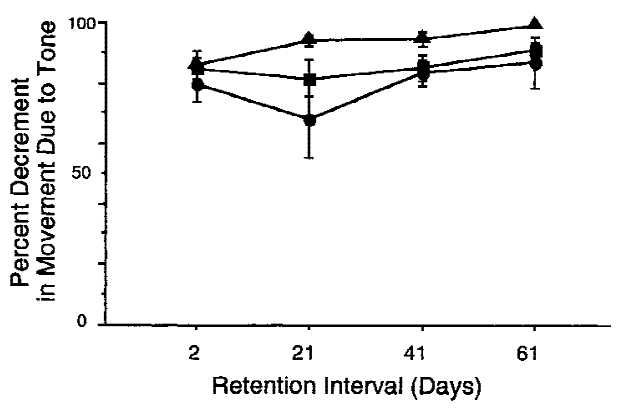

Figure 4: Mean fractional decrement in movement due to the tone, compared to the pre-tone (novel) movement values in each of the three age groups. (O) Young; $(\mathbf{\square})$ adult; $(\boldsymbol{\Lambda})$ old. If one defines the measure of fear as the proportional reduction in movement, these data might be taken as an indication that there was no increase in fear of the tone. Alternatively, this effect could be explained by a nonspecific change in the gain of the circuitry that mediates the expression of the freezing response (i.e., shocked animals may simply become more fearful over time). In this case, it would be unnecessary to postulate changes over time in either the contextual or simple associative memories per se. The present data do not discriminate between these possibilities (see Discussion).

suring performance to a broader spectrum of stimuli for a given learning environment. The present results thus could be interpreted as indicating that there is a decline with age not only in the acquisition and retention of memories that depend initially on the hippocampus, but also in the process by which episodic memory is gradually transformed over time (see Oler and Markus 1998).

At least three different explanations can be suggested for the increased movement suppression over retention interval in the original and novel contexts. One explanation is simply that memory for the context fades with time and hence the animals have increasing difficulty distinguishing the novel from the original environmental contexts. In this view, the rats would express an increasing degree of fear to the novel context because they become less able to discriminate the novel context from the one in which they were originally conditioned. This hypothesis predicts a more rapid increase in fear in the novel context for the old rats, because they show faster forgetting of spatial information (Barnes and McNaughton 1980, 1985), and therefore this idea is inconsistent with the present data. An alternative hypothesis is that there is a gradual strengthening and generalization of the memory of the shock episodes to include not only the immediate shock environment but also the events that led up to the shock or subtle features that were in common to both contexts. These are the sorts of effects that might be anticipated to occur as a result either of repeated actual recurrences of the shock experience or of repeated reactivation of memory traces of the original event (possibly mediated by the hippocampal formation). This is what was observed in the present study, and the data of Oler and Markus (1998) are also consistent with this conclusion.

Before accepting the conclusion that aging results in changes relating to the consolidation process itself, however, it is important to consider an alternative model. The original experience with tone-shock pairing could result in a slow but progressive increase in the gain of the neural circuitry that mediates the suppression of movement in response to fearful stimuli. For example, there might be some nonspecific excitability change taking place over time in amygdala output structures, which could gradually increase the fear response in a manner similar to that observed in the present study. It is known that the amygdala is essential for the expression of these types of conditioned fear behaviors (e.g., Le Doux 1995). At the very least the basal or lateral nuclei are implicated, as selective muscimol injections (Muller et al. 1997) or blockade of NMDA receptors (Lee and Kim 1998) in these nuclei disrupt both the acquisition and expression of the conditioned response. According to this view, there would be no change in the nature of the memory trace with time, rather there is a change in the circuits that transform the memories into behavior. The fact that movement suppression in response to the tone maintains a constant proportionality to the amount of suppression in the novel context alone might be taken as support of the output gain modification hypothesis. If this were correct, then it still might be the case that this change is mediated by hippocampal activity during the retention interval and that the age effects observed here are a result of hippocampal alterations similar to those that appear to underlie age-related changes in spatial memory. This would predict that hippocampal lesions, in addition to blocking the conditioning to the context and its generalization, would also abolish any incubation of the tone-shock association. Moreover, lesions performed after the simple context effect has become independent of the hippocampus (i.e., after about 2 weeks, according to the data of Kim and Fanselow 1992) may nevertheless arrest the continued transformation of that memory over time.

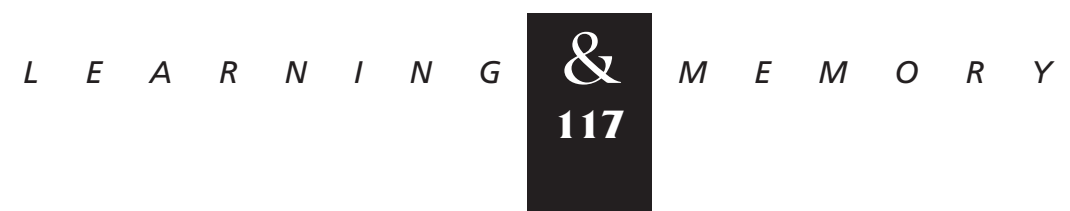


Failure of these predictions would indicate that the incubation effect as a whole is better explained as a progressive change in output gain and that the present age effects reflect a change in this process and not in consolidation per se.

In summary, the present study confirms that there is a decline with age in the transformation over time of memories that normally depend on an intact hippocampus for their initial acquisition and consolidation. It also raises several questions about the nature of the incubation effect and the neural circuits which underlie it.

\section{Acknowledgments}

We thank L. Church, E. Markus, G. Rao, and J. Stoehr for assistance with various aspects of the experimental procedures and apparatus. This work was supported by grant nos. AG03376 from the National Institute on Aging and $\mathrm{MH} 01227, \mathrm{MH} 46823$, and MH01565 from the National Institute of Mental Health.

The publication costs of this article were defrayed in part by payment of page charges. This article must therefore be hereby marked "advertisement" in accordance with 18 USC section 1734 solely to indicate this fact.

\section{References}

Aguado, L., G. Hall, N. Harrington, and M. Symonds. 1998. Illness-induced context aversion learning in rats with lesions of the dorsal hippocampus. Behav. Neurosci.

112: 1142-1151.

Anagnostaras, S.G., S. Maren, and M.S. Fanselow. 1999. Temporally graded retrograde amnesia of contextual fear after hippocampal damage in rats: within-subjects examination. J. Neurosci. 19: 1106-1114.

Barnes, C.A. 1990. Animal models of age-related cognitive decline. In Handbook of neuropsychology (ed. F. Boller and J. Grafman), pp. 169-196. Elsevier Science Publishers, Amsterdam, The Netherlands.

Barnes, C.A. and B.L. McNaughton. 1980. Spatial memory and hippocampal synaptic plasticity in middle-aged and senescent rats. In Psychobiology of aging: Problems and perspectives (ed. D. Stein), pp. 253-272. Elsevier Press/North-Holland, New York, NY.

1985. An age-comparison of the rates of acquisition and forgetting of spatial information in relation to long-term enhancement of hippocampal synapses. Behav. Neurosci. 99: 1040-1048.

Bolhuis, J.J., C.A. Stewart, and E.M. Forrest. 1994. Retrograde amnesia and memory reactivation in rats with ibotenate lesions to the hippocampus or subiculum. Q. J. Exp. Psychol. B Comp. Phys. Psychol. 47: 129-150.

Bolles, R.C. 1970. Species-specific defense reactions and avoidance learning. Psychol. Rev. 77: 32-48.
Buzsáki, G. 1996. The hippocampal-neocortical dialogue. Cereb. Cortex 6: 81-92.

Eichenbaum, H., T. Otto, and N.J. Cohen. 1994. Two functional components of the hippocampal memory system. Behav. Brain Sci. 17: 449-518.

Eysenck, H.J. 1968. A theory of the incubation of anxiety/fear responses. Behav. Res. Ther. 6: 309-321.

Gabriel, M. and J. Vogt. 1972. Incubation of avoidance CRs in the rabbit produced by increase over time in stimulus generalization to apparatus. Behav. Biol. 7: 113-125.

Gage, F.H., S.B. Dunnett, and A. Björklund. 1984. Spatial learning and motor deficits in aged rats. Neurobiol. Aging 5: $43-48$.

Gallagher, M., R. Burwell, and M. Burchinal. 1993. Severity of spatial learning impairment in aging: Development of a learning index for performance in the Morris water maze. Behav. Neurosci. 107: 618-626.

Good, M., L. de Hoz, and R.G.M. Morris. 1998. Contingent versus incidental context processing during conditioning: Dissociation after excitotoxic hippocampal plus dentate gyrus lesions. Hippocampus 8: 147-159.

Karni, A., D. Tanne, B.S. Rubenstein, J.J.M. Askenasy, and D. Sagi. 1994. Dependence on REM sleep of overnight improvement of a perceptual skill. Science 265: 679-682.

Kim, J.J. and M.S. Fanselow. 1992. Modality-specific retrograde amnesia of fear. Science 256: 675-677.

Kim, J.J., R.E. Clark, and R.F. Thompson. 1995. Hippocampectomy impairs the memory of recently, but not remotely, acquired trace eyeblink conditioned responses. Behav. Neurosci. 109: 195-203.

Knowlton, B.J. and M.S. Fanselow. 1998. The hippocampus, consolidation and on-line memory. Curr. Opin. Neurobiol. 8: 193-296.

LeDoux, J.E. 1995. Emotion: Clues from the brain. Annu. Rev. Psychol. 46: 209-235.

Lee, H. and J.J. Kim. 1998. Amygdalar NMDA receptors are critical for new fear learning in previously fear-conditioned rats. J. Neurosci. 18: 8444-8454.

Maren, S. and M.S. Fanselow. 1997. Electrolytic lesions of the fimbria/fornex, dorsal hippocampus, or entorhinal cortex produce anterograde deficits in contextual fear conditioning in rats. Neurobiol. Learn. Mem. 67: 142-149.

Maren, S., G. Aharonov, and M.S. Fanselow. 1997. Neurotoxic lesions of the dorsal hippocampus and Pavlovian fear conditioning in rats. Behav. Brain Res. 88: 261-274.

Marr, D. 1971. Simple memory: A theory for archicortex. Phil. Trans. R. Soc. B. 262: 23-81.

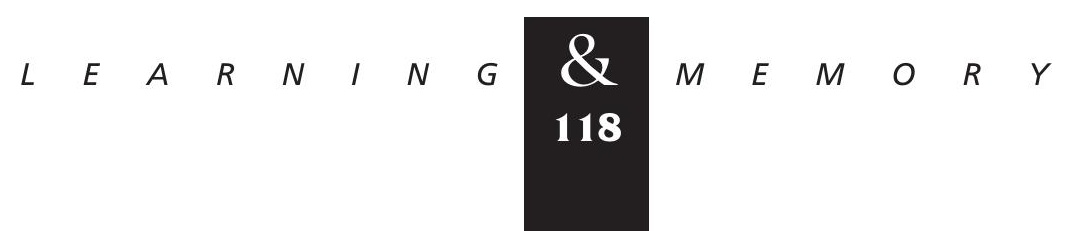


McAllister, V.R. and E.E. McAllister. 1967. Incubation of fear: An examination of the concept. J. Exp. Res. Person. 2: $180-190$.

McClelland, J.L., B.L. McNaughton, and R.C. O'Reilly. 1995. Why are there complementary learning systems in hippocampus and neocortex: Insights from the successes and failures of connectionist models of learning and memory. Psychol. Rev. 102: 419-457.

McCloskey, M. and N.J. Cohen. 1989. Catastrophic interference in connectionist networks: the sequential learning problem. In The psychology of learning and motivation (ed. G.H. Bower), pp. 109-195. Academic Press, New York, NY.

McGaugh, J.L. 1966. Time-dependent processes in memory storage. Science 153: 1351-1358.

McNish, K.A., J.C. Gewirtz, and M. Davis. 1997. Evidence of contextual fear after lesions of the hippocampus: A disruption of freezing but not fear-potentiated startle. J. Neurosci. 17: 9353-9360.

Milner, B. 1972. Disorders of learning and memory after temporal lobe lesions in man. Clin. Neurosurg. 19: 421-426.

Morris, R. 1984. Developments of a water-maze procedure for studying spatial learning in the rat. J. Neurosci. Methods 11: 46-60.

Muller, J., K.P. Corodimas, Z. Fridel, and J.E. LeDoux. 1997. Functional inactivation of the lateral and basal nuclei of the amygdala by muscimol infusion prevents fear conditioning to an explicit conditioned stimulus and to contextual stimuli. Behav. Neurosci. 111: 683-691.

Myers, C.E. and M.A. Gluck. 1994. Context, conditioning, and hippocampal rerepresentation in animal learning. Behav. Neurosci. 108: 835-847.

Nadel, L. and M. Moscovitch. 1997. Memory consolidation, retrograde amnesia and the hippocampal complex. Curr. Opin. Neurobiol. 7: 217-227.

1998. Hippocampal contributions to cortical plasticity. Neuropharmacology 37: 431-439.

Oler, J.A. and E.J. Markus. 1998. Age-related deficits on the radial maze and in fear conditioning: Hippocampal processing and consolidation. Hippocampus 8: 402-415.

Pavlides, C. and J. Winson. 1989. Influences of hippocampal place cell firing in the awake state on the activity of these cells during subsequent sleep episodes. J. Neurosci. 9: 2907-2918.

Phillips, R. and J. LeDoux. 1992. Differential contribution of amygdala and hippocampus to cued and contextual fear conditioning. Behav. Neurosci. 106: 274-285.

Qin, Y.-L., B.L. McNaughton, W.E. Skaggs, and C.A. Barnes. 1997. Memory reprocessing in corticocortical and hippocampocortical neuronal ensembles. Phil. Trans. Royal Soc. B 352: 1525-1533.

Rapp, P.R. and M. Gallagher. 1997. Toward a cognitive neuroscience of normal aging. In Advances in cell aging and gerontology, vol. 2 (ed. P.S. Timrias and E.E. Bitlar), pp. 1-21. JA1 Press Inc., Greenwich, CT.

Scoville, W.B. and B. Milner. 1957. Loss of recent memory after bilateral hippocampal lesions. J. Neurol. Neurosurg. Psychiat. 20: 11-21.

Shen, J. and C.A. Barnes. 1996. Age-related decrease in cholinergic synaptic transmission in three hippocampal subfields. Neurobiol. Aging 17: 439-451.

Skaggs, W.E. and B.L. McNaughton. 1996. Replay of neuronal firing sequences in rat hippocampus during sleep following spatial experience. Science 271: 1870-1873.

Squire, L.R. 1992. Memory and the hippocampus: A synthesis from findings with rats, monkeys, and humans. Psychol. Rev. 99: 195-231.

Squire, L.R. and P. Alvarez. 1995. Retrograde amnesia and memory consolidation: A neurobiological perspective. Curr. Opin. Neurobiol. 5: 169-177.

Squire, L.R., N.J. Cohen, and L. Nadel. 1984. The medial temporal region and memory consolidation: A new hypothesis. In Memory consolidation (ed. E.P.G.

Weingartner), pp. 185-210. Erlbaum, Hillsdale, NJ.

Stoehr, J.D. and G.L. Wenk. 1995. Effects of age and lesions of the nucleus basalis on contextual fear conditioning. Psychobiology 23: 173-177.

Teyler, T.J. and P. DiScenna. 1986. The hippocampal memory indexing theory. Behav. Neurosci. 100: 147-154.

Wilson, M.A. and B.L. McNaughton. 1994. Reactivation of hippocampal ensemble memories during sleep. Science 265: 676-679.

Winocur, G. 1990. Anterograde and retrograde amnesia in rats with dorasal hippocampal or dorsomedial thalamic lesions. Behav. Brain Res. 38: 145-154.

Zola-Morgan, S.M. and L.R. Squire. 1990. The primate hippocampal formation: Evidence for a time-limited role in memory storage. Science 250: 288-289.

Received February 3, 1999; accepted in revised form March 5, 1999.

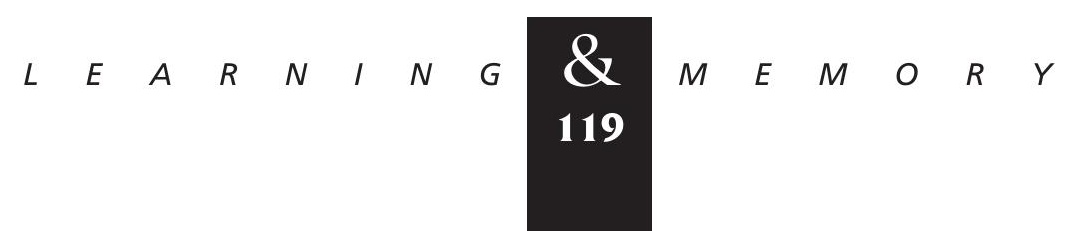




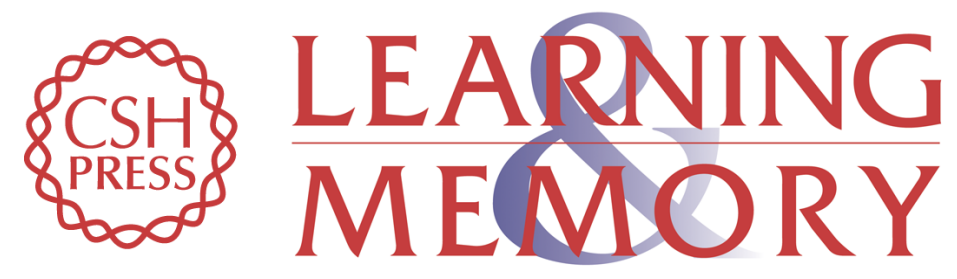

\section{Effects of Age on the Generalization and Incubation of Memory in the F344 Rat}

Frank P. Houston, Gail D. Stevenson, Bruce L. McNaughton, et al.

Learn. Mem. 1999, 6:

Access the most recent version at doi:10.1101//m.6.2.111

References This article cites 40 articles, 10 of which can be accessed free at:

http://learnmem.cshlp.org/content/6/2/111.full.html\#ref-list-1

License

Email Alerting Receive free email alerts when new articles cite this article - sign up in the box at the Service top right corner of the article or click here. 